\title{
Significant resolution of tuberculous pleural effusion on chemotherapy alone
}

\author{
${ }^{1}$ LC Loh, ${ }^{2}$ BK Lim, ${ }^{3} S$ Wan Yusuf \\ ${ }^{1}$ Consultant Chest Physician and Head, Department of Medicine, Penang Medical College, Penang; ${ }^{2}$ Lecturer, Department of Medicine, \\ International Medical University, Kuala Lumpur; ${ }^{3}$ Consultant Physician and Head, Department of Medicine, Tuanku Jaafar Hospital, \\ Seremban, Negeri Sembilan, Malaysia
}

\begin{abstract}
Background: As a standard, significant pleural effusion, whether tuberculous (TB) or not, requires therapeutic thoracocentesis. We tested the hypothesis that standard anti-TB chemotherapy alone can resolve significant pleural effusion. Methods: 20 eligible patients with TB pleural effusion of at least $30 \%$ of the hemithorax ( 10 with moderate-size and 10 with large-size effusion, respectively) were retrospectively reviewed for radiological resolution of their effusions at two, six and 12 months after commencement of standard six-month therapy.

Results: The mean percentage resolutions in both groups were comparable ( $48 \%$ vs $46 \%$ at two months; $59 \%$ vs $85 \%$ at six months; $84 \%$ vs $95 \%$ at 12 months). The mean sizes of effusions were also comparable (I8\% vs $33 \%$ at two months; $14 \%$ vs $10 \%$ at six months; $6 \%$ vs $4 \%$ at 12 months). Cigarette smoking and Indian ethnicity were univariately associated with incomplete resolution of effusions.

Conclusion: Standard anti-TB chemotherapy alone appears to be sufficient to resolve significant TB pleural effusion. An avoidance of therapeutic thoracocentesis may reduce the risk of infection exposure or allow better channelling of resources in certain clinical settings.
\end{abstract}

KEYWORDS Pleural effusion, standard chemotherapy, thoracocentesis, tuberculosis

DECLARATION OF INTERESTS No conflict of interests declared.
Correspondence to LC Loh, Department of Medicine, Penang Medical College, 4 Jalan Sepoy Lines, 10450 Penang, Malaysia

tel. +6042263459

e-mail richard_loh@pmc.edu.my
While the treatment of any pleural effusion involves treating the underlying disease, therapeutic thoracocentesis may be necessary for rapid symptomatic relief and the prevention of complications resulting from inadequate resolution, such as pleural thickening or empyema.' Tuberculous pleural effusion and pleurisy are not uncommon and can occur in up to $30 \%$ of tuberculosis (TB) patients in high prevalence regions of TB and human immunodeficiency virus (HIV). ${ }^{2}$ While diagnostic thoracocentesis is often necessary, the role of therapeutic thoracocentesis in the context of TB has been recently debated, particularly with the advent of effective modern anti-TB chemotherapy regimens.

Some have argued that the frequent occurrence of thickened pleura from TB infection warrants routine therapeutic thoracocentesis, on the assumption that residual pleural fluid increases the risk of the development of pleural thickening with the potential for causing restrictive lung impairment. ${ }^{3,4}$ However, recent studies have also shown that in patients treated with effective anti-TB chemotherapy, therapeutic thoracocentesis performed either once ${ }^{5}$ or repeatedly ${ }^{6}$ to minimise the presence of pleural fluid did not influence the presence or degree of residual pleural thickening or development of restrictive lung impairment. This is likely due to the significant resolution of pleural effusions brought about by effective anti-TB chemotherapy alone.
To test this hypothesis in our own local population of patients, we conducted a retrospective study of non-HIV TB patients with significant pleural effusion (defined as having effusion size $\geq 30 \%$ of the hemithorax) who did not undergo therapeutic thoracocentesis, either at the time of diagnosis or subsequently during the course of standard six-month anti-TB chemotherapy, primarily examining for the percentage resolution and size of pleural effusions at two, six and 12 months after the commencement of chemotherapy, and secondarily for potential clinical-demographic features predictive of incomplete resolution of effusions (defined as having $\geq 5 \%$ of residual pleural effusions/thickening).

\section{PATIENTS AND METHODS}

\section{Data collection}

All medical case notes from the chest clinics of Tuanku Jaafar Hospital, Seremban (available from January 2002), and Port Dickson Hospital, Port Dickson (available from January 2000), Negeri Sembilan, Malaysia, were retrospectively reviewed for eligibility until January 2007. Included were adult patients with confirmed pulmonary TB with pleural effusion treated with a standard anti-TB treatment regimen (two months of daily rifampicin, isoniazid and pyrazinamide with or without ethambutol or streptomycin, followed by four months of a daily or three-times-a-week regimen of rifampicin and isoniazid), 


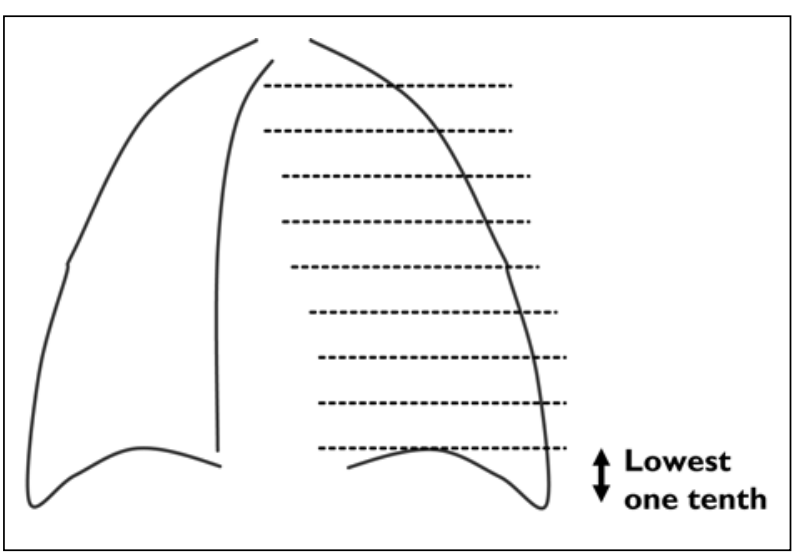

FIGURE I Schematic diagram of the division of a hemithorax into tenths, on which the sizes of pleural effusion are based.

and were followed up for up to one year after the commencement of treatment.

Excluded were: patients in whom the size of pleural effusions was less than $30 \%$ of the hemithorax; patients in whom the TB diagnosis was unconfirmed; HIV patients; patients who received adjuvant corticosteroid therapy; patients with incomplete treatment or follow-up chest $X$-rays; and those whose medical records were incomplete.

Data were collected using a standard questionnaire form based on the information obtained from the standardised TB patient folder used across all government hospitals of the Ministry of Health, Malaysia. Most data that were collected comprised standard information required by the Ministry of Health, except for when thoracocentesis was carried out. For the purpose of this study, we have assumed that thoracocentesis was not done if not recorded.

\section{Outcome measures}

The patients were divided into those with moderatesize effusion (defined as $\geq 30 \%$ to $<50 \%$ of the hemithorax), and those with large-size effusions (defined as $\geq 50 \%$ of the hemithorax). The outcomes were mean percentage resolution of pleural effusions and changes in mean pleural effusion sizes at two, six and 12 months after the initiation of treatment, compared with baseline. The effusion sizes were defined according to tenths of a hemithorax by virtue of the maximum height occupied by the effusions (Figure I).

The first tenth occupies the lowest one-tenth as demarcated at the upper border by an imagery line drawn horizontally at the top of the diaphragm. The other nine-tenths are measured by dividing the rest of the hemithorax first into thirds and then into further thirds. Two investigators (LC Loh and BK Lim) independently evaluated the size of pleural effusions based on posterioanterior views of chest X-ray hard
TABLE I Clinical and demographic characteristics of patients

\begin{tabular}{|c|c|c|c|c|}
\hline \multirow[t]{2}{*}{ Variables } & \multirow{2}{*}{$\begin{array}{l}\text { Entire } \\
\text { group } \\
(n=20)\end{array}$} & \multicolumn{2}{|c|}{ Pleural effusion size } & \multirow[t]{2}{*}{$\mathbf{p}^{* *}$} \\
\hline & & $\begin{array}{l}\text { Moderate } \\
(n=10)\end{array}$ & $\begin{array}{l}\text { Large } \\
(n=10)\end{array}$ & \\
\hline $\begin{array}{l}\text { Mean age, years } \\
\text { (range) }\end{array}$ & $\begin{array}{l}41 \\
(16-70)\end{array}$ & $\begin{array}{l}44 \\
(23-70)\end{array}$ & $\begin{array}{l}38 \\
(|6-6|)\end{array}$ & NS \\
\hline Male & $65 \%$ & $60 \%$ & $70 \%$ & NS \\
\hline $\begin{array}{l}\text { Mean (SD) } \\
\text { weight, kg }\end{array}$ & $52(7.8)$ & $56(8.8)$ & $49(4.7)$ & 0.043 \\
\hline
\end{tabular}

Ethnicity

\begin{tabular}{|l|l|l|l|l|}
\hline Malay & $40 \%$ & $30 \%$ & $50 \%$ & \multirow{2}{*}{ NS } \\
\cline { 1 - 3 } Chinese & $35 \%$ & $40 \%$ & $30 \%$ & \\
\cline { 1 - 3 } Indian & $20 \%$ & $20 \%$ & $20 \%$ & \\
\cline { 1 - 3 } & &
\end{tabular}

Cigarette smokers

\begin{tabular}{|l|l|l|l|l|}
\hline Current & $15 \%$ & $20 \%$ & $10 \%$ & \multirow{2}{*}{ NS } \\
\cline { 1 - 4 } Past & $10 \%$ & $10 \%$ & $10 \%$ & \\
\cline { 1 - 2 } Unknown & $75 \%$ & $70 \%$ & $80 \%$ & \\
\cline { 1 - 3 } & & &
\end{tabular}

Comorbidity scoring*

\begin{tabular}{|c|c|c|c|c|}
\hline Grade I & $55 \%$ & $40 \%$ & $70 \%$ & \multirow[t]{3}{*}{ NS } \\
\hline Grade 2 & $30 \%$ & $40 \%$ & $20 \%$ & \\
\hline Grade 3 & $15 \%$ & $20 \%$ & $10 \%$ & \\
\hline $\begin{array}{l}\text { Mean days with } \\
\text { symptoms (range) }\end{array}$ & $\begin{array}{l}19 \\
(1-156)\end{array}$ & $\begin{array}{l}23 \\
(1-156)\end{array}$ & \begin{tabular}{|l}
14 \\
$(2-52)$
\end{tabular} & NS \\
\hline \multicolumn{5}{|l|}{ Systemic symptoms } \\
\hline Weight loss & $40 \%$ & $30 \%$ & $50 \%$ & NS \\
\hline Fever & $45 \%$ & $40 \%$ & $50 \%$ & NS \\
\hline
\end{tabular}

$\mathrm{SD}=$ standard deviation

*Comorbidity score: ${ }^{13}$ I = no important chronic illness; 2 = moderate/ severe disease of heart, lungs, gastrointestinal tract; 3 = any cancer (except skin), end-stage renal/liver disease

**Comparing between 'moderate' and 'large' size group

films. Patients were also divided into those with complete and incomplete (defined as $\geq 5 \%$ residual pleural effusions/thickening at 12 months) resolution and examined for associations with possible clinical and demographic characteristics.

\section{Statistical analysis}

Descriptive analyses are shown for patients studied. Mean (standard deviation [SD]) percentage resolution and effusion sizes were calculated and compared between the moderate-size and large-size effusion groups for significance in differences using an unpaired t-test at each time point. Continuous and categorical data from the groups of complete and incomplete resolution were studied for potential associations with clinical and demographic characteristics using an unpaired t-test and chi-squared test respectively. $P$ values (two-tailed) of $<0.05$ were considered significant. All statistical analysis was performed using SPSS ${ }^{\mathrm{T}}$ Version $1 \mathrm{I} .0$ for Windows and PrismGraph ${ }^{\mathrm{Tm}}$ Version 3 for Windows. 


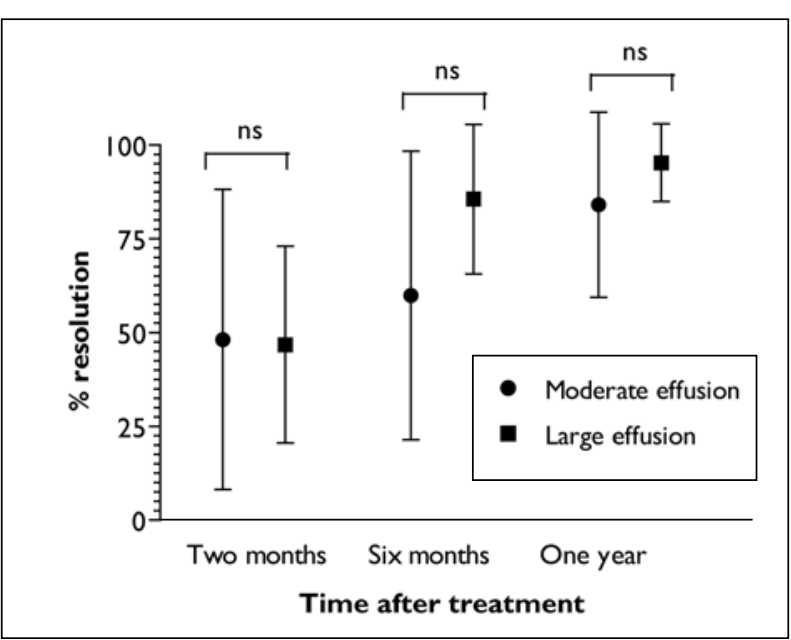

FIGURE 2 Percentage resolution of moderate $(n=10)$ or large $(n=10)$ pleural effusion after standard chemotherapy at two, six and 12 months. Data represented are mean (SD) NS indicates non-significance.

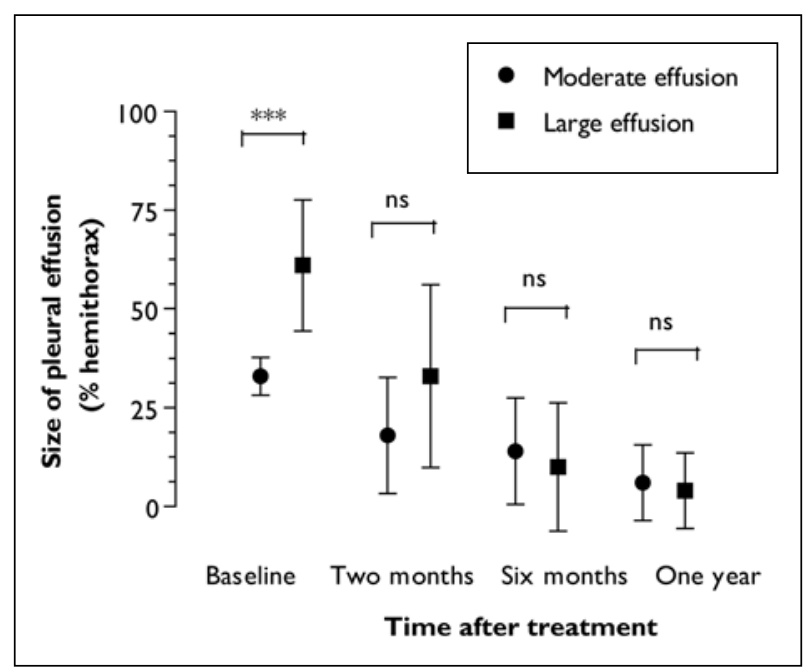

FIGURE 3 Size of pleural effusions from moderate $(n=10)$ or large $(n=10)$ effusion groups at baseline, two, six and 12 months after standard chemotherapy. Data represented are mean (SD). Indicates $\mathrm{P}<0.00 \mathrm{I}$; ns indicates non-significance.

\section{RESULTS}

The case notes of 91 immunocompetent patients with TB pleural effusions who were not treated with therapeutic thoracocentesis were retrieved and reviewed. Of these, 29 had incomplete records (e.g. missing chest $X$-rays) or defaulted follow-up, while another 42 patients had pleural effusions with a size of $<30 \%$ of the hemithorax. Only 20 patients were eligible for the purpose of this study, with 10 patients in each group of moderate-size and large-size effusion. Except for two, all patients had unilateral pleural effusion. The pleural effusion sizes and changes in these two patients were identical on both sides and, as such, their data are analysed as any other patients with unilateral effusions. Patients were well represented for the three main ethnic groups in Malaysia, with the majority being middle-aged
TABLE 2 Clinical and demographic characteristics of patients with complete and incomplete resolution of pleural effusions

\begin{tabular}{|c|c|c|c|}
\hline \multirow[t]{2}{*}{ Variables } & \multicolumn{2}{|c|}{ Resolution at I 2 months } & \multirow[t]{2}{*}{$\mathbf{p}^{* *}$} \\
\hline & $\begin{array}{l}\text { Complete } \\
(n=15)\end{array}$ & $\begin{array}{l}\text { Incomplete } \\
(n=5)\end{array}$ & \\
\hline Mean age, years (range) & $4 \mathrm{I}(23-70)$ & $44(|6-6|)$ & NS \\
\hline Male & $60 \%$ & $80 \%$ & NS \\
\hline Mean (SD) weight, kg & $5 I(5.9)$ & $56(11.9)$ & NS \\
\hline \multicolumn{4}{|l|}{ Ethnicity } \\
\hline Malay & $40 \%$ & $40 \%$ & \multirow[t]{3}{*}{0.046} \\
\hline Chinese & $46 \%$ & $0 \%$ & \\
\hline Indian & $6.7 \%$ & $60 \%$ & \\
\hline \multicolumn{4}{|l|}{ Cigarette smokers } \\
\hline Current & $13 \%$ & $20 \%$ & \multirow[t]{3}{*}{0.027} \\
\hline Past & $0 \%$ & $40 \%$ & \\
\hline Unknown & $87 \%$ & $40 \%$ & \\
\hline \multicolumn{4}{|l|}{ Comorbidity scoring* } \\
\hline Grade I & $60 \%$ & $40 \%$ & \multirow[t]{3}{*}{ NS } \\
\hline Grade 2 & $33 \%$ & $20 \%$ & \\
\hline Grade 3 & $7 \%$ & $40 \%$ & \\
\hline $\begin{array}{l}\text { Mean days with } \\
\text { symptoms (range) }\end{array}$ & II (I-52) & $32(I-156)$ & NS \\
\hline \multicolumn{4}{|l|}{ Systemic symptoms } \\
\hline Weight loss & $46 \%$ & $20 \%$ & NS \\
\hline Fever & $46 \%$ & $40 \%$ & NS \\
\hline
\end{tabular}

$\mathrm{SD}=$ standard deviation

* Comorbidity score: ${ }^{13} \mathrm{I}$ = no important chronic illness;

2 = moderate/severe disease of heart, lungs, gastrointestinal tract;

$3=$ any cancer (except skin), end-stage renal/liver disease

** Comparing between 'complete' and 'incomplete' group

and male with some chronic medical conditions (Table I). Except for a lower weight average in the large-size effusion group, all other clinical and demographic characteristics were comparable.

Mean percentage resolutions of pleural effusion in both groups were comparable and not significantly different: $48 \%$ vs $46 \%$ at two months; $59 \%$ vs $85 \%$ at six months; $84 \%$ vs $95 \%$ at 12 months (Figure 2). Initial mean sizes of pleural effusion in moderate- and large-size effusion were significantly different $(33 \%$ vs $61 \%, p<0.001)$. At two, six and 12 months the mean sizes of effusion between both groups had reduced and were comparable: $18 \%$ vs $33 \%$; $14 \%$ vs $10 \%$; $6 \%$ vs $4 \%$, p $>0.05$ (Figure 3 ). Overall at 12 months, the mean percentage resolution of all effusion was $89.7 \%$, with a mean effusion size of $5 \%$.

Comparing those with complete $(n=15)$ and incomplete resolution $(n=5)$, Indian ethnicity and history of cigarette smoking were univariately associated with incomplete resolution, although it must be acknowledged that smoking data were missing for 15 patients $(\mathrm{p}=0.046$ and 0.027 respectively) (Table 2 ). 


\section{DISCUSSION}

We have shown that TB pleural effusion of significant size (defined as $\geq 30 \%$ of the hemithorax) can undergo significant resolution on standard six-month anti-TB chemotherapy alone by 12 months. The findings were similar whether the effusions were moderate or large. By the end of the two-month intensive phase of anti-TB therapy, the resolution of the pleural effusion was almost $50 \%$, and by 12 months' follow-up the overall mean size of pleural effusion was only $5 \%$. In the five patients defined as having incomplete resolution, their mean pleural size was only $8 \%$; this group was univariately associated with cigarette smoking and Indian ethnicity.

The sample size of eligible patients turned out to be small because most patients with our definition of significant pleural effusions had already undergone therapeutic thoracocentesis. Because this was a retrospective study highly dependent on the accuracy of medical records and availability of X-ray hard films, there were also many patients who were ineligible because of the lack of relevant data. However, the cohort could conveniently be divided equally into those with moderateand large-size effusions, permitting some meaningful analysis. Furthermore, we were careful to examine a sample that is relatively homogenous without serious confounding factors, such as having prescribed oral corticosteroids or infection with HIV, which are frequently encountered in TB patients today.

The findings of our small retrospective study support the proposition that the current standard six-month anti-TB chemotherapy is effective at resolving TB pleural effusions of significant size, and are consistent with the conclusions of some controlled trials. In a prospective study of six months' duration, Lai et al..$^{5}$ compared 30 patients with TB pleural effusions that were initially treated with pigtail catheter drainage with another 3 I patients treated by anti-TB chemotherapy alone, and showed that the frequency of residual pleural thickening was similarly low with no significant impact on lung function. In another prospective study, Hoheisel et al. ${ }^{6}$ examined 16 patients who had repeated pleural tapping over a four-week period, compared with another 16 patients on anti-TB treatment alone. They also showed that at the end of four weeks the percentage of patients with absent pleural effusions was $63 \%$ and $44 \%$ respectively, and at the end of four months no patients had pleural effusions or even minimal residual changes with the exception of one patient who required surgical pleurectomy for significant pleural thickening. Both studies concluded that therapeutic thoracocentesis offers no additional benefit to standard anti-TB treatment and that the development of residual pleural thickening does not appear to be influenced by such intervention.

It is important to point out that our study did not seek to clarify whether the pleural effusions were loculated or not. A recent study by Chung et al. ${ }^{7}$ shows that early pigtail catheter drainage with intrapleural thrombolysis may benefit those with loculated TB pleural effusions, compared with those with free-flowing pleural effusions. Their findings suggest that there may be a role for early therapeutic thoracocentesis if pleural effusions are loculated, and therefore differentiation between loculated or free-flowing effusions should be made early during the course of management.

We are unclear as to the clinical relevance of Indian ethnicity and cigarette smoking in the development of residual pleural effusion/thickening, although the detrimental effects of cigarette smoking on airway mucociliary function and the association of cigarette smoking with higher risks for smear-positive, cavitating upper lobe TB disease are well documented..$^{8,9}$ The association of lower body weight with the large-size effusion group may be explained by the presence of a more aggressive and prolonged TB infection with consequent greater body mass loss. ${ }^{10}$ In our cohort of patients, the size of residual effusion/thickening is small and very unlikely to cause any restrictive lung impairment of clinical relevance. However, we had no formal lung function testing results to prove or refute this.

The implication of our findings, together with other existing evidence, is relevant in today's clinical practice. Tuberculosis remains a serious contagious infection that requires proper infection control measures. The handling of respiratory secretions, particularly aerosolised sputum," should be minimised and therefore the avoidance of unnecessary contact from prolonged or repeated thoracocentesis is probably advisable. The same holds true in certain resource-challenged areas where the knowledge that therapeutic thoracocentesis may not always be necessary may be helpful in allowing better channelling of medical resources and funding. The latter is particularly relevant in today's healthcare era where the combat of TB remains in the hands of poorerresourced countries. ${ }^{12}$

Acknowledgment The authors wish to thank the support of the chest clinics' staff in Tuanku Jaafar Hospital, Seremban, and Port Dickson Hospital, Malaysia, in the conduct of the study, as well as the kind permission of the Director-General, the Ministry of Health, Malaysia, to use the data. 


\section{REFERENCES}

I Rahman NM, Chapman SJ, Davies RJ. Diagnosis and management of infectious pleural effusion. Treat Respir Med 2006; 5:295-304. doi:10.2165/00151829-200605050-00001

2 Ferrer Sancho J. Pleural tuberculosis: incidence, pathogenesis, diagnosis and treatment. Curr Opin Pulm Med 1996; 2:327-34.

3 Large SE, Levick RK. Aspiration in the treatment of primary tuberculous pleural effusion. BMJ 1958; I:I5I2-4. doi:10.1136/ bmj.I.5086.15I2

4 Morrone N, Lombardi MC, Machado O. Prevention of pleural thickening through pleural aspiration in patients with tuberculous effusion.J Pneumol (Sao Paulo) 1989; I5:180-4.

5 Lai YF, Chao TY,Wang YH et al. Pigtail drainage in the treatment of tuberculous pleural effusions: a randomised study. Thorax 2003; 58: I49-5I. doi:I0.II36/thorax.58.2.149

6 Hoheisel G, Vogtmann M, Chan KS et al. [Pleuritis tuberculosa therapeutic value of repeated chest tapping.] Pneumologie 2004; 58:23-7. In German. doi:10.1055/s-2003-8I2437

7 Chung $\mathrm{CL}$, Chen $\mathrm{CH}$, Yeh $\mathrm{CY}$ et al. Early effective drainage in the treatment of loculated tuberculous pleurisy. Eur Respir J 2008; 31:I26I-7. doi: I0.1 183/09031936.00122207
8 Wanner A. A review of the effects of cigarette smoke on airway mucosal function. Eur J Respir Dis Suppl 1985; 139:49-53.

9 Chiang CY, Slama K, Enarson DA. Associations between tobacco and tuberculosis. Int J Tuberc Lung Dis 2007; I I:258-62.

10 Lönnroth K,Williams BG, Cegielski P et al. A consistent log-linear relationship between tuberculosis incidence and body mass index. Int J Epidemiol 2010; 39:149-55. doi:10.1093/ije/dyp308

II Jensen PA, Lambert LA, lademarco MF et al. Guidelines for preventing the transmission of Mycobacterium tuberculosis in healthcare settings. Atlanta: National Center for HIV, STD, and TB Prevention, CDC; 2005. Available from: http://www.cdc.gov/tb/ publications/guidelines/infectioncontrol.htm

12 World Health Organization. Global tuberculosis control - a short update to the 2009 report. Geneva:WHO; 2009. Available from: http://www.who.int/tb/en/

I3 Brancati FL, Chow JW,Wagener MM et al. Is pneumonia really the old man's friend? Two-year prognosis after community-acquired pneumonia. Lancet 1993; 342:30-3. doi:I0.I016/0|40-6736(93)9|887-R

\section{DATES FOR YOUR DIARY: FORTHCOMING SYMPOSIA}

Yorkshire symposium: Recent advances

in medicine (Bradford Royal Infirmary) 25 June

\begin{tabular}{ll}
\hline - Care of the elderly & 15 September \\
\hline Paediatric symposium & \\
(RCPE/RCPCH joint symposium) & 23 September \\
\hline
\end{tabular}

- Diabetes and endocrinology:

learning from other specialists

7 October

Collegiate Members' symposium: updates in internal medicine

22 October

Neurology

27 October

- Renal medicine

(SRA/RCPE joint event)

5 \& 6 November

Gastroenterology

II November

Cardiology

23 November

50th St Andrew's Day Festival: updates in acute medicine
All symposia are held at the Royal College of Physicians of Edinburgh unless otherwise stated.

Programme details will become available at: http://events.rcpe.ac.uk

Contact the Education Assistant (tel: 0131 225 7324, email: h.elliott@rcpe.ac.uk) to be added to the mailing list for an event.

Unable to attend a particular symposium? Selected lectures (more than 100 currently available) can be listened to in the Online Education section of the secure area of the College website. Log on at http://www.rcpe.ac.uk. If you have lost or forgotten your username or password, please contact webmaster@rcpe.ac.uk.

Online booking is now available at http://events.rcpe.ac.uk 